\title{
GENE THERAPY FOR LUNG CANCER: ENHANCEMENT OF TUMOR SUPPRESSION BY A COMBINATION OF SEQUENTIAL SYSTEMIC CISPLATIN AND ADENOVIRUS-MEDIATED P53 GENE TRANSFER
}

Dao M. Nguyen, MD, MSc, FRCSC $^{\mathrm{a}}$

Francis R. Spitz, MD

Nancy Yen, MSc ${ }^{\mathrm{a}}$

Richard J. Cristiano, $\mathrm{PhD}^{\mathrm{a}}$

Jack A. Roth, MD, FACS ${ }^{\mathrm{a}}$

\begin{abstract}
A more effective gene therapy strategy for lung cancer using sequential cisplatin administration and adenovirus-mediated p53 gene transfer was developed on the basis of our previous observation of enhanced expression of a reporter gene in malignant cells exposed to cisplatin before gene transfer. Transfer of the normal (wildtype) $\mathbf{p 3 3}$ gene into cisplatin-treated H1299 cells, in which p53 is homozygously deleted, resulted in up to a $60 \%$ further inhibition of cell proliferation in vitro than p53 transfer into untreated $\mathrm{H1299}$ cells. The cisplatin plus p53 gene transfer strategy yielded significantly greater apoptosis and tumor growth suppression in an animal model of subcutaneous H1299 tumor nodules than wildtype p53 gene transfer alone. The timing of cisplatin administration and p53 gene transfer was shown to be critical: cisplatin administration simultaneous with or subsequent to p53 gene transfer was less effective than cisplatinfirst sequential treatment. Moreover, the in vivo inhibition of tumor growth was maintained by repeated cycles of treatment. This gene therapy strategy has been incorporated into a phase I clinical trial for the treatment of lung cancer and provides a basis for the development of improved therapeutic protocols. (J Thorac Cardiovasc Surg 1996;112:1372-7)
\end{abstract}

$\mathrm{M}$ utations of the p53 tumor suppressor gene have been found in up to $60 \%$ of non-small-cell lung carcinomas (NSCLC). ${ }^{1}$ Restoration of normal p53 function in cells with p53 mutations by gene replace-

From the Section of Thoracic Molecular Oncology, Departments of Thoracic and Cardiovascular Surgery, ${ }^{a}$ Department of Surgical Oncology, ${ }^{\mathrm{b}}$ The University of Texas M. D. Anderson Cancer Center, Houston, Tex.

This study was partially supported by grants from the National Cancer Institute and National Institutes of Health (R01 CA45187 [J.A.R.]; R29 CA66037 [R.J.C.]); by gifts to the Division of Surgery from Tenneco and Exxon for the Core Lab Facility; by the M.D. Anderson Cancer Center Core Support Grant (CA16672); by a grant from the Mathers Foundation; and by a sponsored research agreement from Introgen Therapeutics, Inc., Houston, Tex.

Read at the Seventy-sixth Annual Meeting of The American Association for Thoracic Surgery, San Diego, Calif., April 28-May 1, 1996.

Received for publication May 6, 1996; revisions requested June 10, 1996; revisions received July 11, 1996; accepted for publication July 15, 1996.

Address for reprints: Jack A. Roth, MD, The University of Texas M. D. Anderson Cancer Center, Department of Thoracic and Cardiovascular Surgery, Box 109, 1515 Holcombe Blvd., Houston, TX 77030.

Copyright (C) 1996 by Mosby-Year Book, Inc.

$0022-5223 / 96 \$ 5.00+0 \quad \mathbf{1 2 / 6 / 7 6 5 0 8}$ ment leads to $G_{1}$ cell cycle arrest or induction of apoptosis. $^{2}$ A recombinant adenovirus expressing the p53 gene (Adv-p53) has been shown to mediate tumor growth inhibition both in vitro and in vivo., However, in some cases malignant cells may eventually resume growth, some at the same rate as untreated cells, after completion of gene transfer., $\mathrm{We}^{5}$ previously demonstrated that a brief exposure of NSCLC cells to the chemotherapeutic agent cisplatin (cisdiamminodichloroplatinum; CDDP) before adenovirus-mediated gene transfer enhanced target cell expression of a reporter gene in vitro and in vivo. In this study, we exploited this salutary effect of CDDP on gene expression by designing a novel gene therapy strategy for lung cancer that comprises sequential CDDP administration and adenovirusmediated p53 gene transfer. We hypothesized that the resulting increased $\mathrm{p} 53$ gene expression in target cells may lead to an enhanced tumoricidal effect.

\section{Materials and methods}

Cell proliferation assay. H1299 cells (human NSCLC cells with a homozygous deletion of p53) were exposed to CDDP at a concentration of $0.062 \mu \mathrm{g} / \mathrm{ml}$ of medium for 24 hours. Forty-eight hours later, cells were infected with Adv-p53 at a multiplicity of infection (MOI: number of 
viral particles per cell) of 1 or 5 . Daily cell counts were performed for 5 days after infection. Controls consisted of untreated cells, cells exposed to CDDP only, cells infected with Adv-p53 only, and cells infected with dI312 (E1Adeleted adenovirus used as control vector) with or without prior CDDP treatment.

In situ TUNEL assay for apoptosis. Terminal deoxynucleotidyl transferase-mediated dUTP-biotin nick endlabeling (TUNEL) assay was performed on CDDPtreated or control H1299 cells 12 hours and 24 hours after infection with Adv-p53 and on cells of the control groups (as described earlier) at the same time points (MOI $=5$ ). ${ }^{6}$ The fractions of TUNEL-positive cells were determined by flow cytometry (EPICS Profile II, Coulter Corp., Hialeah, Fla.). In situ TUNEL assay was also performed on frozen tissue sections of tumor nodules harvested 24 hours after intratumoral injections with Adv-p53 or d1312. ${ }^{6}$ Positive controls were normal cells or tissue sections treated with DNAse I (Gibco-BRL, Gaithersburg, Md.).

In vivo combination of sequential CDDP and Adv-p53. H1299 tumor xenografts were established in the subcutaneous spaces of nu/nu mice. An intraperitoneal CDDP injection ( $5 \mu \mathrm{g} / \mathrm{gm}$ body weight) was given on day 0 followed by an intratumoral injection of $5 \times 10^{9} \mathrm{Adv}-\mathrm{p} 53$ particles in $100 \mu \mathrm{l}$ of phosphate-buffered saline solution (PBS) on days 2, 4, and 6. Other CDDP and Adv-p53 combinations were also studied for their tumoricidal efficacy: simultaneous intraperitoneal CDDP and Advp53 administration in three equally divided doses (CDDP: $1.67 \mu \mathrm{g} / \mathrm{gm}$ body weight) on days 2,4 , and 6 or intraperitoneal CDDP ( $5 \mu \mathrm{g} / \mathrm{gm}$ body weight) given on day 8 (2 days after completion of three intratumoral Adv-p53 injections on days 2, 4, and 6). In two other groups of mice, a second cycle of gene therapy was given 10 days after completion of the first cycle to study the therapeutic efficacy of repeated therapy. Tumor sizes were measured every 2 days for 32 days, and tumor volumes were estimated by assuming a spherical shape with the average tumor diameter calculated as the square root of the product of the orthogonal diameters; $n=5$ per group.

All animals received humane care in compliance with the "Principles of Laboratory Animal Care" formulated by the National Society for Medical Research and the "Guide for the Care and Use of Laboratory Animals" prepared by the Institute of Laboratory Animal Resources and published by the National Institutes of Health.

\section{Results and discussion}

Treatment with either CDDP or infection with d1312 with or without prior CDDP exposure had no effect on H1299 cell growth in vitro. However, H1299 cells exposed to a low concentration of CDDP $(0.062 \mu \mathrm{g} / \mathrm{ml}) 48$ hours before Adv-p53 infection had a $31 \%$ to $60 \%$ greater inhibition of cell proliferation than cells infected only with Adv-p53. This inhibition was apparent as early as 3 days after gene transfer at MOI of 1 and 5, respectively (Fig. 1). This combination strategy also resulted in a

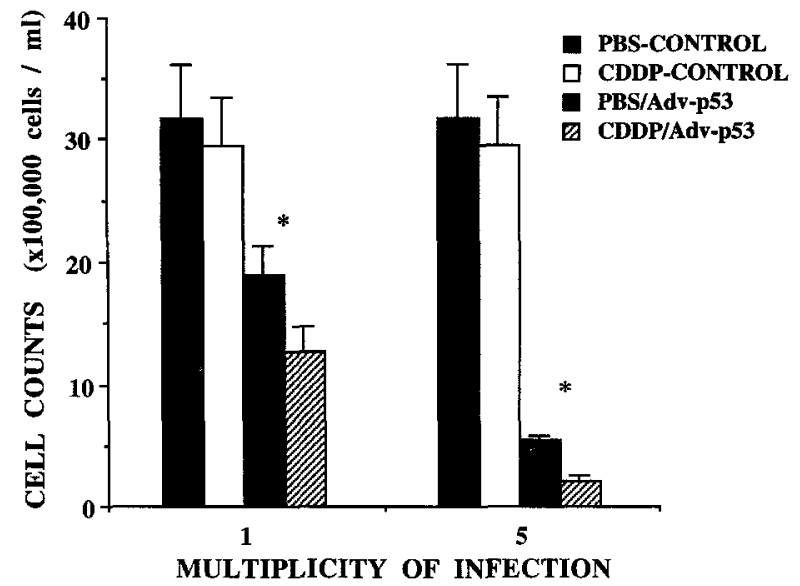

Fig. 1. Enhancement of the tumor killing effect by the combination of CDDP and Adv-p53 in vitro ( ${ }^{*} p<0.01$ vs PBS/Adv-p53 by Student's $t$ test). Cells counts performed on day 3 after Adv-p53 infection are shown here $(n=5)$.

higher level of p53 protein expression at $12,24,36$, and 48 hours after virus infection than in cells infected with Adv-p53 alone as determined by a quantitative Western blot analysis as described elsewhere. ${ }^{7}$ Densitometry analysis showed that the relative levels of p53 protein in CDDP-treated cells (normalized for $\beta$-actin levels) were twofold to threefold higher than in Adv-p53-infected cells without prior CDDP exposure (data not shown). Although fewer than $5 \%$ of cells in other control groups stained positive for apoptosis by TUNEL assay, $19.2 \%$ and $68.5 \%$ of cells infected with Advp53 only $(\mathrm{MOI}=5)$ were TUNEL-positive at 12 hours and 24 hours after p53 gene transfer. The fractions of apoptotic cells were significantly higher (50.2\% and $90.8 \%$ at the same time points) in cells treated by the combination of CDDP and Adv-p53. The higher levels of p53 gene expression achieved by this combination gene transfer strategy may enhance induction of apoptosis, which would translate to increased inhibition of tumorigenicity.

In the in vivo studies, intraperitoneal administration of CDDP resulted in only a slightly greater reduction in tumor growth (Fig. 2, $A$ ). Tumors injected with $\mathrm{dl} 312$, with or without prior intraperitoneal CDDP, showed some degree of growth retardation. Intratumoral injections of Adv-p53 without CDDP inhibited tumor growth during and immediately after the treatment (Fig. 2, $A$ ), but these tumors resumed growth and attained a mean tumor 

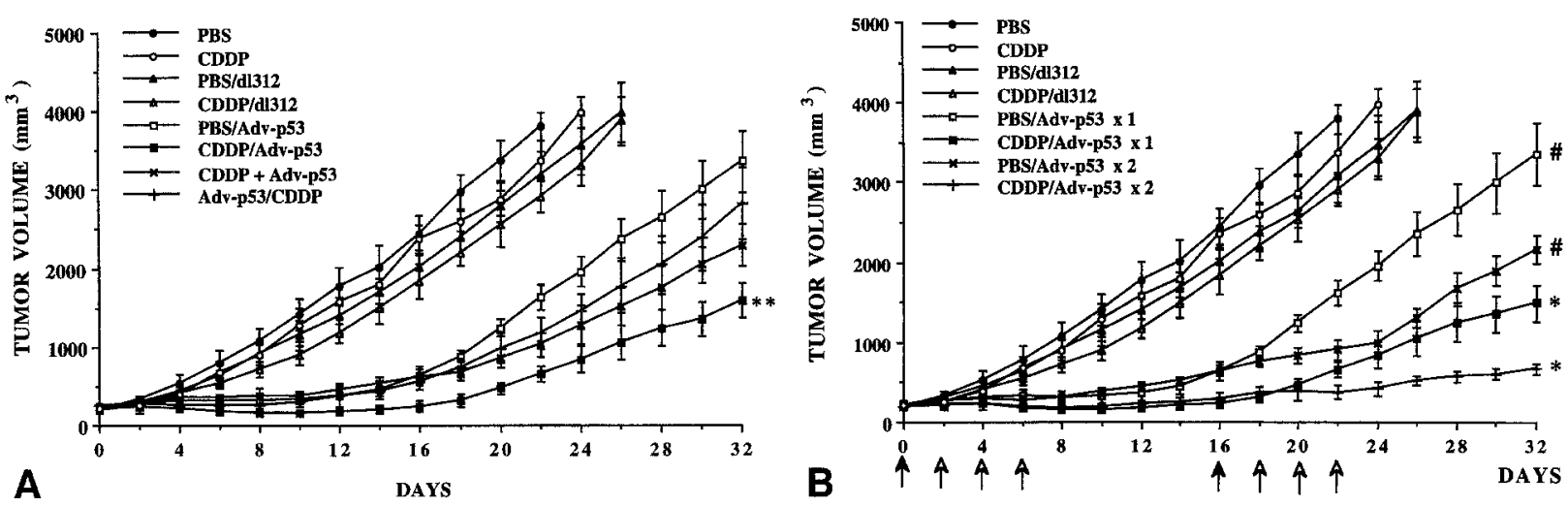

Fig. 2. Enhanced in vivo inhibition of tumorigenesis by sequential intraperitoneal CDDP and intratumoral Adv-p53 injections. A, Analysis of timing of CDDP administration with intraperitoneal CDDP given either before, concurrent (CDDP + Adv-p53) with, or after (Adv-p53/CDDP) intratumoral injections of Adv-p53 ${ }^{* *} p<0.01 \mathrm{CDDP} / \mathrm{Adv}$-p53 vs other groups by analysis of variance and Student's $t$ test). B, Analysis of the effect of a second course of gene therapy. The symbols \# and * denote PBS and CDDP groups, respectively (\#, ${ }^{*} p<0.01$ vs one cycle of therapy by Student's $t$ test). For both experiments, the controls were tumor-bearing animals injected with PBS (PBS), Adv-p53 without prior intraperitoneal CDDP (PBS/Adv-p53), or d1312 with or without prior intraperitoneal CDDP (CDDP/dl312 or $\mathrm{PBS} / \mathrm{dl} 312$ ).

volume of $3357 \pm 391 \mathrm{~mm}^{3} 32$ days after the onset of therapy. However, the combination of intraperitoneal CDDP gene therapy resulted in a pronounced inhibition of tumor development: at the end of the observation period, the average tumor size was $1497 \pm 221 \mathrm{~mm}^{3}(p<0.01$ vs Adv-p53 without prior CDDP). Systemic CDDP administration before 553 gene replacement therapy, therefore, produced a synergistic tumor growth inhibition effect that was responsible for at least a $55.4 \%$ further reduction in tumor size. Moreover, the timing of intraperitoneal CDDP administration was of critical importance, inasmuch as the sequential combination of CDDP and Adv-p53 showed a more significant tumor growth inhibitory effect than the other two dosing schedules (Fig. 2, A). A second cycle of gene therapy further inhibited tumorigenesis. Of the groups that received a second cycle, the CDDP-treated group had a significantly higher degree of tumor growth suppression (54.6\%) than the PBS-treated group (35.7\%), and both groups had significantly higher degrees of tumor growth suppression than the respective groups receiving a single cycle of therapy (Fig. 2, B). An extensive induction of apoptosis was noted in tumors treated by sequential $\mathrm{CDDP} / \mathrm{Adv}$-p53 therapy as compared with those treated with Adv-p53 only or controls
(Fig. 3). The magnitude of tumor growth suppression achieved by the other treatment regimens (CDDP given during or after Adv-p53 injections) was more than a simple addition of tumoricidal effects attributable to either CDDP or Adv-p53 alone. In these cases, transduction of wildtype p53 to the p53-negative cells may have made these cells more susceptible to CDDP, an observation reported by Fujiwara and associates. ${ }^{6}$ The gene therapy strategy presented in this report, which involves a CDDP-induced enhancement of p53 gene expression and elevated p53-mediated cell arrest or apoptosis, shows the highest degree of tumor growth inhibition.

We have developed a new, more effective gene therapy strategy using the sequential combination of CDDP administration and adenovirus-mediated p53 gene transfer for the treatment of NSCLC. This is a particularly attractive and clinically applicable cancer gene therapy strategy for several reasons: (1) p53 mutations are quite prevalent in many types of cancer; (2) CDDP-induced enhancement of gene expression has been observed in other malignant cells of different tissue origins, ${ }^{5}$ and (3) CDDP is a commonly used chemotherapeutic agent. This gene therapy strategy is now being tested for lung cancer in a phase I clinical trial at our institution. 

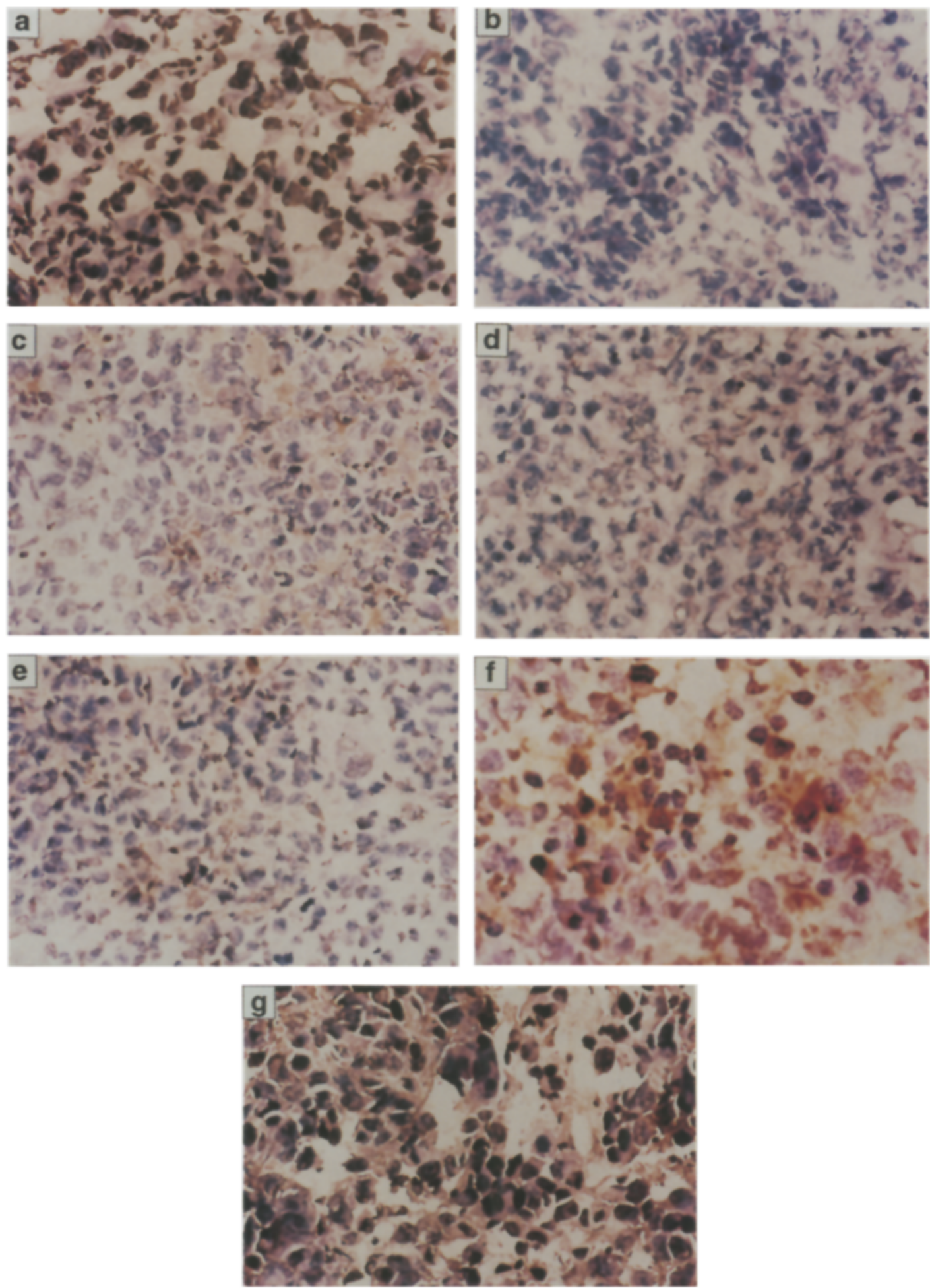

Fig. 3. Extensive induction of apoptosis in H1299 tumors treated with the combination of sequential intraperitoneal CDDP and intratumoral Adv-p53 injections as analyzed by the in situ TUNEL assay. Treatments: A, positive control; B, normal; C, intraperitoneal CDDP; D, PBS/dl312; E, CDDP/dl312; F, PBS/Adv-p53; G, CDDP/Adv-p53. Nuclei of apoptotic cells containing fragmented DNA are stained brown. 


\section{REFERENCES}

1. Chiba I, Takahashi T, Nau MN, et al. Mutations in the p53 gene are frequent in primary, resected non-small cell lung cancer. Oncogene 1990;5:1603-10.

2. Liu TJ, Zhang WW, Taylor DL, et al. Growth suppression of human head and neck cancer cells by the introduction of a wildtype p 53 gene via a recombinant adenovirus. Cancer Res 1994;54:3662-7.

3. Wills KN, Maneval DC, Menzel P, et al. Development and characterization of recombinant adenoviruses encoding human p53 for gene therapy of cancer. Hum Gene Ther 1994;5: 1079-88.

4. Eastham JA, Hall SJ, Sehgal I, et al. In vivo gene therapy with p53 or p21 adenovirus for prostate cancer. Cancer Res 1995; 55:5151-5.

5. Nguyen DM, Spitz F, Kataoka M, et al. Enhancement of gene transduction in human carcinoma cells by DNA-damaging agents. Proc Am Assoc Cancer Res 1996;37:347.

6. Fujiwara T, Grimm EA, Mukhopadhyay T, et al. Induction of chemosensitivity in human lung cancer cells in vivo by adenoviral-mediated transfer of the wildtype p53 gene. Cancer Res 1994;54:2287-2291.

7. Zhang WW, Fang X, Mazur W, et al. High efficiency gene transfer and high-level expression of wild-type p53 in human lung cancer cells mediated by recombinant adenovirus. Cancer Gene Ther 1994;1:5-13.

\section{Discussion}

Dr. Larry R. Kaiser (Philadelphia, Pa.). Were you injecting your adenoviral construct into visible tumor nodules? How long had your tumor nodules been growing subcutaneously in the nude animals before you injected them?

Dr. Nguyen. It took about 3 weeks to achieve a volume of $250 \mathrm{~mm}^{3}$.

Dr. Kaiser. And you were using irradiated nude mice as well. Does this tumor not grow in nude mice that have not been irradiated?

Dr. Nguyen. It would take about 5 weeks to grow in unirradiated nude mice.

Dr. Kaiser. Did you look at transgene persistence as well? You did some reinjection studies, I guess the thought being that you may have looked at transgene persistence. It was not particularly long, so you reinjected, which gets into some additional questions that we can talk about as well.

Dr. Nguyen. When we study p53 immunohistochemical staining or apoptosis beyond 24 or 48 hours, usually the cells have already gone to apoptosis and we could not detect it. However, when we use a reporter gene like $\beta$-galactosidase, we could detect expression of the gene up to 10 days or even longer.

Dr. Kaiser. You did your reinjection how many days after the frst injection?

Dr. Nguyen. This was 10 days after the last injection; that means 10 days after completion of the gene therapy protocol.

Dr. Kaiser. Did you look then at persistence after the second injection as well, because even in these nude animals, there is still some cellular immune response? Was your transgene persistence attenuated with the second injection?
Dr. Nguyen. No, we did not look at that. We haven't made a detailed analysis of the growth curve after the second cycles, but to us it appeared that the second cycle is not as strong as the first cycle. It might be related to the tumor size at the second cycle of injections. As you know, once the tumor develops and the number of cells increase, we would use the same number of viral particles, and the MOI would decrease.

Dr. Kaiser. It seems that you are suppressing growth over a period of time, but is it just a time-related phenomenon? It does seem that even after the second injection, you still are seeing some increase in growth in the nodule. Have you looked at any other ways of combining therapy to try to attenuate that?

Dr. Nguyen. Yes.

Dr. Kaiser. You have used a cell line that has a deleted p53. Have you evaluated any lines that have a mutated p53 to see if you get the same results putting in the wildtype p53 construct?

Dr. Nguyen. To answer your second question first: In terms of the status of p53, we used a deleted p53 cell line. However, all the studies in our laboratory and others have demonstrated that the effects should be the same with a mutated p53. We use this to give us an advantage so that we can detect the wildtype p53 expression more easily. Otherwise we have to use a marker, for example, flag p53, to detect $\mathrm{p} 53$ expression in cells that express mutated $\mathrm{p} 53$. To answer that question, we have not done the experiment, but in vitro data indicated that it should give the same effects whether we have mutated or deleted p53.

To answer the first question, our goal is to try to eradicate a tumor. We can use a combination of CDDP followed by Adv-p53 gene transfer followed by CDDP or irradiation, because there are existing experimental data to indicate that restoration of wildtype p53 status can sensitize malignant cells having deleted or mutated p53 gene to subsequent external beam irradiation or systemic chemotherapy.

Dr. Kaiser. Have you looked at this in a syngeneic model that is immunocompetent? The biggest question with adenoviral gene therapy is the immune response. Adenovirus stimulates quite an effective immune response, certainly from the first injection, and then the question arises about rechallenging these animals that now are no longer naive.

Dr. Nguyen. No, we have not evaluated this in a syngeneic model. The vector is always a thorny issue for gene therapists, and work is being done in that field. I do think that in an immunocompetent host the tumor suppression effect is probably better. Recent work indicates that a tumor-suppression effect can be induced with $\beta$-galactosidase alone because of the inducing of anti- $\beta$ galactosidase $\mathrm{T}$ cells.

Dr. Kaiser. That probably is not the case. In fact, the immune system seems to work very definitely against this, as we have shown. The persistence of the transgene is so short, especially when rechallenged, that it seems to be much more of a problem than any of us had anticipated.

My other question concerns the bystander effect. Initially your group talked about a bystander effect with p53 and then we never heard anything else about it. Have you done any more work with the bystander effect? 
Dr. Nguyen. Yes, we are still working on that, but it is not as prominent as we thought it could be. It has been well demonstrated in mixed experiments that there was a bystander effect when the retroviral vector was used with p53, and we have seen some results, but the effect is not as concrete as with the HSV-TK system that you have been working with.

Dr. Jhingook Kim (Seoul, Korea). I have some questions that may be similar to Dr. Kaiser's questions. First, how do you decide the amount of the CDDP in your experiment? Just with CDDP, we sometimes see apoptosis and growth suppression in the cultured lung cancer cell lines. What is your experimental base to choose that dose of the CDDP?

My second question concerns the mechanism of the improved effectiveness. Do you think the improvement of your gene therapy is due to the increased infection rate or increased cytocidal effects of the delivered p53?

My third question is similar to Dr. Kaiser's question. Why do you apply your second dose of gene therapy at that time? Are you afraid of the immunity of the adenovirus if you use it after growth of the tumor?

Dr. Nguyen. To answer your question about the dose and the schedules, that stemmed from our previous study using the $\beta$-galactosidase gene as the reporter gene, and we did dose-response studies as well as time studies. We demonstrated maximum enhancement only at this dose. This is not a tumor-killing dose. At this dose there is no change in cell growth characteristic. We did the studies in vivo as well, and there is no evidence of apoptosis at this dose. This dose somehow stimulated cells to express the gene better. It is not a phenomenon of increased uptake of viral particles. We did studies as well, and we demonstrated that there is an increased expression of the successfully transfused viral particles.

The dose was derived from studies that we did with the $\beta$-galactosidase gene. Similarly, for in vivo studies we demonstrated an enhanced expression of the $\beta$-galactosidase gene 2 and 4 days after infection when we used a dose of $5 \mu \mathrm{g} / \mathrm{gm}$ of body weight. That is why we used this dose and that is why we used these time schedules.

It is not clear why CDDP, which damages deoxyribonucleic acid (DNA), produces this kind of enhancement effect. We have seen that with other drugs as well that have a DNA-damaging effect, such as VP-16, and we have seen that with irradiation as well. It must have something to do with damage to DNA, DNA repair, and probably it creates a microenvironment that is favorable for DNA expression. There is thus an increase in expression of the gene rather than an increased uptake of viral particles.

I agree with you about immune reactions. That is going to be a big issue for gene therapists. We just want to see whether having a second-cycle therapy in our model can suppress tumor growth further. If it is going to be a problem in the syngeneic model, then our impetus would be to kill the tumor in the first attempt. That is the whole idea of our experiment, which is to enhance the tumorkilling effect. 